\title{
Post-Prior discrepancies in CDW-EIS calculations for ion impact ionization fully differential cross sections
}

\author{
M F Ciappina and $\mathrm{W} R$ Cravero \\ CONICET and Departamento de Física, Av. Alem 1253, (8000) Bahía Blanca, \\ Argentina \\ E-mail: ciappina@uns.edu.ar
}

\begin{abstract}
.
In this work we present fully differential cross sections (FDCSs) calculations using post and prior version of CDW-EIS theory for helium single ionization by $100 \mathrm{MeV} \mathrm{C} \mathrm{C}^{6+}$ $\mathrm{amu}^{-1}$ and $3.6 \mathrm{MeV} \mathrm{amu}^{-1} \mathrm{Au}^{24+}$ and $\mathrm{Au}^{53+}$ ions. We performed our calculations for different momentum transfer and ejected electron energies. The influence of internuclear potential on the ejected electron spectra is taken into account in all cases. We compare our calculations with absolute experimental measurements. It is shown that prior version calculations give better agreement with experiments in almost all studied cases.
\end{abstract}

Submitted to: J. Phys. B: At. Mol. Phys. 


\section{Introduction}

The study of electron emission spectra in ion atom collisions has been a field of intense activity for years (Stolterfoht et al 1997). For intermediate to high energy single ionization there has been considerable theoretical efforts focused in the so-called two centre electron emission (TCEE) (Fainstein et al 1991, Pedersen et al 1990). Improvement in the description of the ionized electron moving in the presence of both residual target and projectile fields after the collision (final state) has been key for the correct description of experimental data (Gulyás et al 1995).

Within distorted wave approximations, it has been shown that, at least for high impact energy and multiply charged projectiles, CDW theory of Belkić (1978) used together with an appropriate description of the initial bound and final continuum electron states, yields best results for doubly differential cross sections (DDCSs) (Gulyás and Fainstein 1998, Ciappina et al 2003). However, when the projectile impact velocity decreases, the CDW-EIS theory of Crothers \& McCann (1983) gives better results, its only difference being the choice of the initial state. Moreover CDW-EIS approximation is formally free of criticisms regarding the initial state proper normalization, and the transition amplitudes have not the divergent behavior that CDW exhibits (Crothers 1982) (although it has been demonstrated that CDW amplitudes are integrable and its DDCSs are well behaved) (Dubé and Dewangan 1995).

The field has experienced a renewed interest as a result of the development of the experimental technique known as COLTRIMS (cold-target recoil-ion momentum spectroscopy) (Moshammer et al 1994). With COLTRIMS, the projectile's tiny scattering angle can be obtained indirectly by measuring the ionized electron and recoil ion momenta. Fully differential cross sections (FDCS) for ion impact ionization can be measured now and this constitute a challenging ground for existing theories (Foster et al 2004).

The first measurements of the FDCS, for various momentum transfers and ejectedelectron energies, were reported in 2001 by Schulz et al for single ionization of helium by $100 \mathrm{MeV} \mathrm{amu}^{-1} \mathrm{C}^{6+}$. Theoretical results for this process were made later by Madison et al 2002, using several approximation schemes. They obtained reasonable good agreement between experiment and theory in the scattering plane for intermediate momentum transfer, but the theories used was not able to reproduce the measurements for large values of momentum transfer and out of scattering plane.

Subsequently, experiments with other projectiles and energy ranges have been performed. Fischer et al (2003) have reported absolute experimental measurements for $2 \mathrm{MeV} \mathrm{amu}^{-1} \mathrm{C}^{6+}$ single ionization of helium in the scattering plane for various momentum transfers and ejected-electron energies. Foster et al (2004) have presented 3DW-EIS results for the single ionization of helium by $3.6 \mathrm{MeV} \mathrm{amu}^{-1} \mathrm{Au}^{24+}$ and $\mathrm{Au}^{53+}$ ions. The 3DW-EIS model is a modified version of the CDW-EIS approximation

and, although the authors found good agreement with $2 \mathrm{MeV} a m u^{-1} \mathrm{C}^{6+}$ data, the theory did not yield a significant improvement for higher charged ions. Theoretical 
results calculated using a CDW-EIS model exhibited differences between experiment and theory on an absolute scale for emission in the scattering plane, defined by the plane containing the initial and final projectile momenta. Their calculations were made using a post version of the CDW-EIS theory and an active electron approximation with hydrogenic wavefunctions for the initial and final electron states. Indeed, the simplest description for the He bound initial state is to assume it has one 'active' and one 'passive' electron and that the 'active' electron can be described as moving in the effective Coulomb field of the atomic core with an effective charge chosen either: (a) to reproduce the ionization energy or (b) so that the continuum wave is orthogonal to the initial state.

A more sophisticated description involves the use of full numerical Hartree-Fock wave functions for both initial and final states of the active electron (Gulyás et al 1995, Gulyás and Fainstein 1998, Foster et al 2004). Hartree-Fock description, however, does not include proper angular correlations in the initial state, and for large perturbations, there might be the chance that the projectile interacts with more than one electron in a single event. An explicit two-electron description, i.e., a full-blown four-body theory for the collision process might be necessary in that case. We have shown that by using the prior version of CDW-EIS together with an appropriate Roothan-Hartree-Fock description of the initial state and an effective charge coulomb wave function for the target - electron continuum, we get similar results to those obtained by using numerical Hartree-Fock wave functions (Ciappina et al 2004), for ion impact helium ionization DDCSs.

The aim of this paper is to present post and prior CDW-EIS calculations with internuclear interaction between the projectile and the target ( $\mathrm{N}-\mathrm{N}$ interaction) taken into account for ion helium single ionization FDCSs at different perturbation regimes. Atomic units are used throughout unless otherwise stated.

\section{Theories}

We regard He single ionization as a single electron process and assume that (i) the initial state for the 'active' electron is described by a semi-analytical Rothan-HartreeFock scheme using a 5 parameters wave function (Clemente and Roetti 1974) and (ii) in the final state the 'active' target electron moves in the combined Coulomb field of the target core with an effective charge $Z_{e f f}=1.6875$. The electron-projectile relative motion are represented in a CDW-EIS approach, i.e. one eikonal phase in the entrance channel and a pure Coulomb distortion in the final one. $\mathrm{N}-\mathrm{N}$ interaction is treated as a pure Coulomb interaction between the projectile with a charge $Z_{P}$ and the true target core charge, $Z_{T}=1$.

$\mathrm{N}-\mathrm{N}$ interaction is taken into account in the transition amplitude $a_{i f}(\boldsymbol{\rho})$, in the usual semi-classical or eikonal approximation, through its multiplication by a phase factor (McCarroll and Salin 1978), which for pure coulomb internuclear interaction results in 
(Crothers and McCann 1983)

$$
a_{i f}^{\prime}(\boldsymbol{\rho})=\mathrm{i}(\rho v)^{2 \mathrm{i} \nu} a_{i f}(\boldsymbol{\rho})
$$

were $\nu=Z_{P} Z_{T} / v, v$ is the velocity of the impinging projectile and $\rho$ is the impact parameter $(\boldsymbol{\rho} \cdot \mathbf{v}=0)$. $a_{i f}(\boldsymbol{\rho})\left(a_{i f}^{\prime}(\boldsymbol{\rho})\right)$ is the transition amplitude with (without) internuclear interaction. Using two-dimensional Fourier transforms we have for the transition amplitude elements, CDW-EIS transition matrix can be written alternatively as a function of the momentum transfer:

$$
T_{i f}^{\prime}\left(\boldsymbol{\eta}^{\prime}\right)=\frac{\mathrm{i} v^{2 \mathrm{i} \nu}}{(2 \pi)^{2}} \int d \boldsymbol{\eta} T_{i f}(\boldsymbol{\eta}) \int d \boldsymbol{\rho} \rho^{2 \mathrm{i} \nu} e^{\mathrm{i}\left(\boldsymbol{\eta}-\boldsymbol{\eta}^{\prime}\right) \cdot \boldsymbol{\rho}} a_{i f}(\boldsymbol{\rho})
$$

We solve the integral over impact parameter analytically to obtain:

$$
T_{i f}^{\prime}(\boldsymbol{\eta})=\nu \frac{\mathrm{i} v^{2 \mathrm{i} \nu}(2 \pi)^{-\mathrm{i} \nu}}{2^{4} \pi^{3}} \int d \boldsymbol{\eta}^{\prime} T_{i f}\left(\boldsymbol{\eta}^{\prime}\right)\left|\boldsymbol{\eta}-\boldsymbol{\eta}^{\prime}\right|^{-2(1+\mathrm{i} \nu)}
$$

The remaining integral in (3) is evaluated numerically with an adaptive integration scheme. This approximation is valid as long as (i) the projectile suffers very small deflections in the collision and (ii) the velocity of the recoil ion remains small compared to that of the emitted electron.

Within CDW-EIS, Transition amplitude can be computed as

$$
T_{i f}^{+C D W-E I S}=\left\langle\chi_{f}^{-C D W}\left|W_{f}^{\dagger}\right| \chi_{i}^{+E I S}\right\rangle
$$

in its post version or

$$
T_{i f}^{-C D W-E I S}=\left\langle\chi_{f}^{-C D W}\left|W_{i}\right| \chi_{i}^{+E I S}\right\rangle
$$

in the prior version, where the initial (final) state distorted wave $\chi_{i}^{+}\left(\chi_{f}^{-}\right)$is an approximation to the initial (final) state which satisfies outgoing-wave $(+)$ (incomingwave $(-))$ conditions. For the initial state the asymptotic form of the Coulomb distortion (eikonal phase) is used in the electron-projectile interaction together with a semi analytical Rothan-Hartree-Fock description for the initial bound-state wavefunction (Clementi and Roetti 1974)

$$
\chi_{i}^{+E I S}=(2 \pi)^{-3 / 2} \exp \left(\mathrm{i} \mathbf{K}_{i} \cdot \mathbf{R}_{T}\right) \psi_{1 s}\left(\mathbf{r}_{T}\right) \mathcal{E}_{v}^{+}\left(\mathbf{r}_{P}\right)
$$

where $\mathcal{E}_{v}^{+}\left(\mathbf{r}_{P}\right)$ is

$$
\mathcal{E}_{v}^{+}\left(\mathbf{r}_{P}\right)=\exp \left(-\mathrm{i} \frac{Z_{P}}{v} \ln \left(v r_{P}-\mathbf{v} \cdot \mathbf{r}_{P}\right)\right) .
$$

The final state wavefunction is collected into the form (Rosenberg 1973, Garibotti and Miraglia 1980, Crothers and McCann 1983)

$$
\chi_{f}^{-C D W}=(2 \pi)^{-3 / 2} \exp \left(\mathrm{i} \mathbf{K}_{f} \cdot \mathbf{R}_{T}\right) \chi_{T}^{-}\left(\mathbf{r}_{T}\right) C_{P}^{-}\left(\mathbf{r}_{P}\right)
$$

where $C_{P}^{-}$represents the Coulomb distortion of the ejected electron wave function due to the projectile coulomb potential.

$$
C_{P}^{-}\left(\mathbf{r}_{P}\right)=N\left(\nu_{P}\right){ }_{1} F_{1}\left(-\mathrm{i} \nu_{P}, 1,-\mathrm{i} k_{P} r_{P}-\mathrm{i} \mathbf{k}_{P} \cdot \mathbf{r}_{P}\right)
$$


being $\nu_{P}=\frac{Z_{P}}{k_{P}}$ the Sommerfeld parameter, and $\mathbf{k}_{P}$ is the relative momentum of the e-P subsystem. The ${ }_{1} F_{1}$ is the Kummer function and $N\left(\nu_{P}\right)$ is the usual normalization factor

$$
N\left(\nu_{P}\right)=\Gamma\left(1-\mathrm{i} \nu_{P}\right) \exp \left(-\pi \nu_{P} / 2\right)
$$

being $\Gamma$ the gamma function. On the other hand $\chi_{T}^{-}\left(\mathbf{r}_{T}\right)$ is the wave function for the ejected electron in the field of the target residual ion.

$$
\chi_{T}^{-}\left(\mathbf{r}_{T}\right)=(2 \pi)^{-3 / 2} \exp \left(\mathrm{ik}_{T} \cdot \mathbf{r}_{T}\right) N\left(\nu_{T}\right)_{1} F_{1}\left(-\mathrm{i} \nu_{T}, 1,-\mathrm{i} k_{T} r_{T}-\mathrm{i} \mathbf{k}_{T} \cdot \mathbf{r}_{T}\right)
$$

being $\nu_{T}=\frac{Z_{T}}{k_{T}}$ and now $\mathbf{k}_{T}$ is the relative momentum of the e-T subsystem. We use $Z_{T}=Z_{\text {eff }}=1.6875$ to model the screened target residual ion as a pure Coulomb potential.

The perturbation potentials $W_{f}$ in equation (4) and $W_{i}$ in (5) are defined by

$$
\left(H_{f}-E_{f}\right) \chi_{f}^{-}=W_{f} \chi_{f}^{-}
$$

and

$$
\left(H_{i}-E_{i}\right) \chi_{i}^{+}=W_{i} \chi_{i}^{+}
$$

where $H_{f}\left(H_{i}\right)$ are the full electronic final (initial) Hamiltonian (neglecting the total center of mass motion) and $E_{f}\left(E_{i}\right)$ are the total final (initial) energy of the system in the $\mathrm{cm}$ frame respectively.

The explicit forms of these operators can be written (Crothers and Dubé 1992)

$$
W_{f}=-\nabla_{\mathbf{r}_{T}} \cdot \nabla_{\mathbf{r}_{P}}
$$

and

$$
W_{i}=\frac{1}{2} \nabla_{\mathbf{r}_{P}}^{2}-\nabla_{\mathbf{r}_{T}} \cdot \nabla_{\mathbf{r}_{P}}
$$

In the centre of mass frame, the FDCS in energy and ejection angle of the electron, and direction of the outgoing projectile is given by (Berakdar et al 1993, Inokuti 1971, Bethe 1930)

$$
\frac{\mathrm{d}^{3} \sigma}{\mathrm{d} E_{k} \mathrm{~d} \Omega_{k} \mathrm{~d} \Omega_{K}}=N_{e}(2 \pi)^{4} \mu^{2} k \frac{K_{f}}{K_{i}}\left|T_{i f}\right|^{2} \delta\left(E_{f}-E_{i}\right)
$$

where $N_{e}$ is the number of electrons in the atomic shell, $\mu$ is the reduced mass of the projectile-target subsystem, $K_{i}\left(K_{f}\right)$ is the magnitude of the incident particle initial (final) momentum. The ejected-electron's energy and momentum are given by $E_{k}$ and $k$ respectively. The solid angles $\mathrm{d} \Omega_{K}$ and $\mathrm{d} \Omega_{k}$ represent the direction of scattering of the projectile and the ionized electron, respectively. We use non-orthogonal Jacobi coordinates $\left(\mathbf{r}_{P}, \mathbf{r}_{T}\right)$ to outline the collision process. These coordinates are the position of the active electron with respect to the projectile $\left(\mathbf{r}_{P}\right)$ and to the target ion $\left(\mathbf{r}_{T}\right)$ respectively. Also the coordinate $\mathbf{R}_{T}$ is needed, that represents the position of the incoming projectile with respect to the center of mass of the subsystem e- $\mathrm{T}$. If we neglect terms of order $1 / M_{T}$ and $1 / M_{P}$, where $M_{T}$ is the mass of the target ion nucleus and $M_{P}$ is the corresponding to the incident heavy ion, we can write $\mathbf{R}_{T}=\mathbf{r}_{T}-\mathbf{r}_{P}$. 
We have replaced the transition matrix in the post and prior schemes (equations (4) and (50) in the definition of FDCS (16) and we have applied it to several single ionization processes.

\section{Results}

We have performed calculations for different projectiles, spanning a large range of perturbation strengths as measured by charge to velocity ratio $\eta=Z_{P} / v$. In figure 1 we present results for $100 \mathrm{Mev} \mathrm{amu}^{-1} \mathrm{C}^{6+}$ (Schulz et al 2001) single ionization of Helium calculated in prior CDW-EIS, for different values of electron emission energy $\left(E_{e}\right)$ and momentum transfer $\left(\mathbf{q}=\mathbf{K}_{i}-\mathbf{K}_{f}\right)$. Calculations are in very good agreement with available experimental results.

In figure 2 we layout results for other theories applied to the same process for an intermediate value of electron energy and momentum transfer. We see that prior CDW-EIS gives the best results. Even when $\eta=0.1$ both FBA and post CDW-EIS fail to accurately describe the experimental results, although they broadly reproduce the angular distribution.

Figures 3 and 4 show results for $3.6 \mathrm{MeV} \mathrm{amu}^{-1} \mathrm{Au}^{24+}$ impact ionization of $\mathrm{He}$ (Fischer et al 2003), calculated in prior and post CDW-EIS. For $E_{e}=4.0 \mathrm{eV}$, results for prior version are in reasonable agreement with experiment, However both theories fail to correctly reproduce the strong forward emission peak, which is due to the strong projectile electron post collisional interaction (PCI). Trend is similar for $E_{e}=10.0 \mathrm{eV}$, (Figure 4) where we see a better performance in prior version calculations, in particular in the prediction of the direct peak position. Note that no renormalization factor is included in these calculations.

In figures 5 and 6 we show prior and post CDW-EIS calculations for $3.6 \mathrm{MeV} \mathrm{amu}^{-1}$ $\mathrm{Au}^{53+}$ impact ionization of He. Even when we are stretching the validity range of the perturbative treatment a bit too much $\left(\eta \approx 2,4.4\right.$ for $\mathrm{Au}^{24+}$ and $\mathrm{Au}^{53+}$ projectiles respectively), angular structure with only one strong peak is correctly predicted in prior version while post version of the theory predicts two distinct direct and recoil peak. However the position of the peak is not correctly given in prior version, again because the theory underestimates the strong PCI between the impinging ion and the ejected electron, which shifts the emission towards the forward direction. Both versions including $\mathrm{N}-\mathrm{N}$ interaction fail to yield the correct order of magnitude of experimental data. Large projectile charges are likely to induce quite a large polarization in the target. Effective charges both for residual target-electron and $\mathrm{N}-\mathrm{N}$ interactions are probably not the same than for lower charged projectiles, and it is indeed very probable that the effective charge approach is not a good approximation here. Model potentials taking into account polarization effects need to be considered for the target, at least in the exit channel, but most probably in both initial and final states. 


\section{Conclusions}

We have performed FDCSs calculations for highly charged ion impact ionization of Helium. We employed prior and post versions of CDW-EIS theories taken into account $\mathrm{N}-\mathrm{N}$ interaction but otherwise using as simple an approach for electronic wave functions as possible. Indeed, use of prior version helps us to avoid the need of more precise wave functions for the initial or final electronic state. We found reasonably good agreement with experimental data, even for projectile charges for which the system is arguably outside the range of validity of a perturbative theory.

We see that for emission in the collision plane, three body dynamics seems to be enough to explain most of the structures observed for low energy emission and low projectile charge. For $\mathrm{Au}^{24+}$ and $\mathrm{Au}^{53+}$ projectiles the larger emission in the forward direction is not well reproduced by the theory but, as said before, those cases are outside the range where perturbative treatments are known to be valid. However, if the effect of target polarization in the entrance channel and the inclusion of higher orders in the exit channel distortions, are taken into account, perturbation based calculations could probably be brought closer to experimental results.

\section{Acknowledgments}

This work has been partially supported by Consejo Nacional de Investigacones Científicas y Técnicas, Argentina, ANPCYT, PICT and Universidad Nacional del Sur under PGI 24/F027. One of us (MFC) is grateful for the hospitality of the Max Planck Institut für Kernphysik in Heidelberg.

\section{References}

Bethe H 1930 Ann. Phys., Lpz 5325

Belkić Dž 1978 J. Phys. B: At. Mol. Phys. 113529

Berakdar J, Briggs J S and Klar H 1989 J. Phys. B: At. Mol. Opt. Phys. 26285

Ciappina M F, Cravero W R and Garibotti C R 2003 J. Phys. B: At. Mol. Opt. Phys. 363775

Ciappina M F, Cravero W R and Garibotti C R 2004 Phys. Rev. A 70062713

Clemente E and Roetti C 1974 At. Data Nucl. Data Tables 14177

Crothers D S F 1982 J. Phys. B: At. Mol. Phys. 152061

Crothers D S F and McCann J F 1983 J. Phys. B: At. Mol. Phys. 163229

Crothers D S F and Dubé L J 1992 Adv. At. Mol. Opt. Phys. 30 287-337

Dubé L J and Dewangan D P 1995 19th Int. Conf. on Physics of Electronic and Atomic Collisions (Whistler) Abstracts p 62

Fainstein P D, Ponce V H and Rivarola R D 1991 J. Phys. B: At. Mol. Opt. Phys. 243091

Fainstein P D and Gulyás L J. Phys. B: At. Mol. Opt. Phys. 38 (2005) 317

Fischer D, Moshammer R, Schulz M, Voitkiv A and Ullrich J 2003 J. Phys. B: At. Mol. Opt. Phys. 36 3555

Foster M, Madison D H, Peacher J L, Schulz M, Jones S, Fischer D, Moshammer R and Ullrich J 2004 J. Phys. B: At. Mol. Opt. Phys. 371565

Garibotti C R and Miraglia J E 1980 Phys. Rev. A 21572 
Gulyás L, Fainstein P D and Salin A 1995 J. Phys. B: At. Mol. Opt. Phys. 28245

Gulyás L and Fainstein P D 1998 J. Phys. B: At. Mol. Opt. Phys. 313297

Inokuti M 1971 Rev. Mod. Phys. 43297

McCarroll R and Salin A 1978 J. Phys. B: At. Mol. Opt. Phys. 11 L693

Moshammer R, Ullrich J, Unverzagt M, Schmitt W, Jardin P, Olson R E, Mann R, Dörner R, Mergel V, Buck U and Schmidt-Böcking H 1994 Phys. Rev. Lett. 733371

Pedersen J O, Hvelplund P, Petersen A G and Fainstein P D 1990 J. Phys. B: At. Mol. Opt. Phys. 23 L597

Rosenberg L 1973 Phys. Rev. D 81833

Schulz M, Moshammer R, Madison D H, Olson R E, Marchalant P, Whelan C T, Walters H R J, Jones S, Foster M, Kollmus K, Cassimi A and Ullrich J 2001 J. Phys. B: At. Mol. Opt. Phys. 34 L305

Stolterfoht N, DuBois R D and Rivarola R D 1997 Electron Emission in Heavy Ion-Atom Collisions (Springer:Berlin) 


\section{Figure captions}

Figure 1. FDCS for $100 \mathrm{MeV} \mathrm{amu}^{-1} \mathrm{C}^{6+}$ single ionization of Helium calculated in prior CDW-EIS: solid line; experimental data, (Schulz et al 2001) solid circles. (a) $E_{e}=6.5 \mathrm{eV},|q|=0.88$ a.u. (b) $E_{e}=17.5 \mathrm{eV},|q|=1.43$ a.u. (c) $E_{e}=37.5 \mathrm{eV}$, $|q|=2.65$ a.u.

Figure 2. FDCS for $100 \mathrm{MeV} \mathrm{amu}^{-1} \mathrm{C}^{6+}$ single ionization of Helium calculated for $E_{e}=17.5 \mathrm{eV}$ and $|q|=1.43$ a.u. in prior CDW-EIS: solid line; post CDW-EIS: dashed line; FBA: dotted line; experimental data: (Schulz et al 2001) solid circles.

Figure 3. FDCS for $3.6 \mathrm{MeV} \mathrm{amu}{ }^{-1} \mathrm{Au}^{24+}$ single ionization of Helium for $E_{e}=4 \mathrm{eV}$. Prior CDW-EIS: solid line; post CDW-EIS: dashed line; experimental data: (Fischer et al 2003) solid circles. Note that the angle of electron emission has been changed with respect to the other figures and now is measured in a range of $-180^{\circ}$ to $+180^{\circ}$, being $0^{\circ}$ the direction of the incoming projectile.

Figure 4. Same as in figure 3 for $E_{e}=10 \mathrm{eV}$.

Figure 5. FDCS for $3.6 \mathrm{MeV} \mathrm{amu}{ }^{-1} \mathrm{Au}^{53+}$ single ionization of Helium for $E_{e}=4 \mathrm{eV}$. Prior CDW-EIS: solid line; post CDW-EIS: dashed line; experimental data: (Fischer et al 2003) solid circles.

Figure 6. Same as in figure 5 for $E_{e}=10 \mathrm{eV}$. 


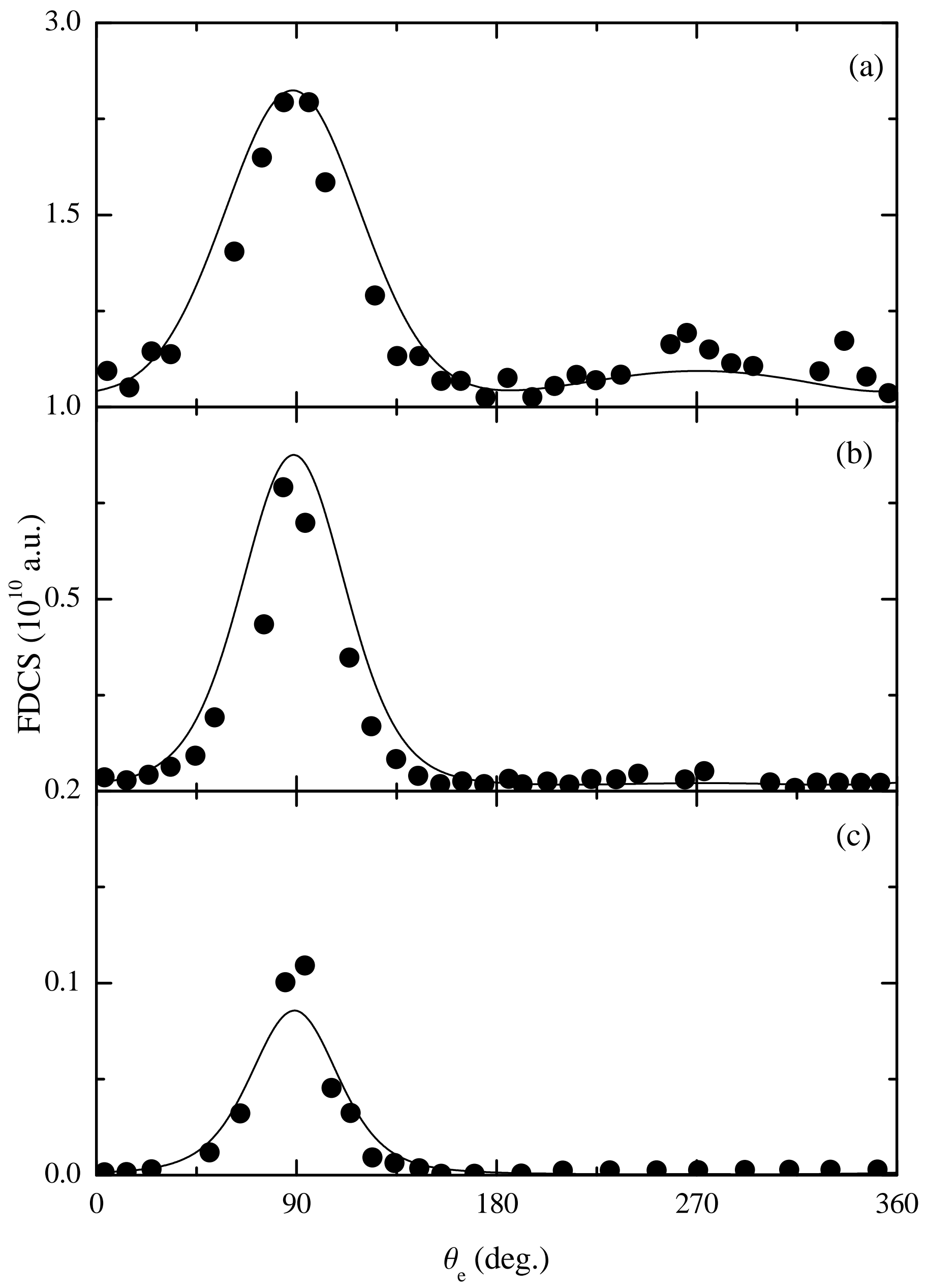




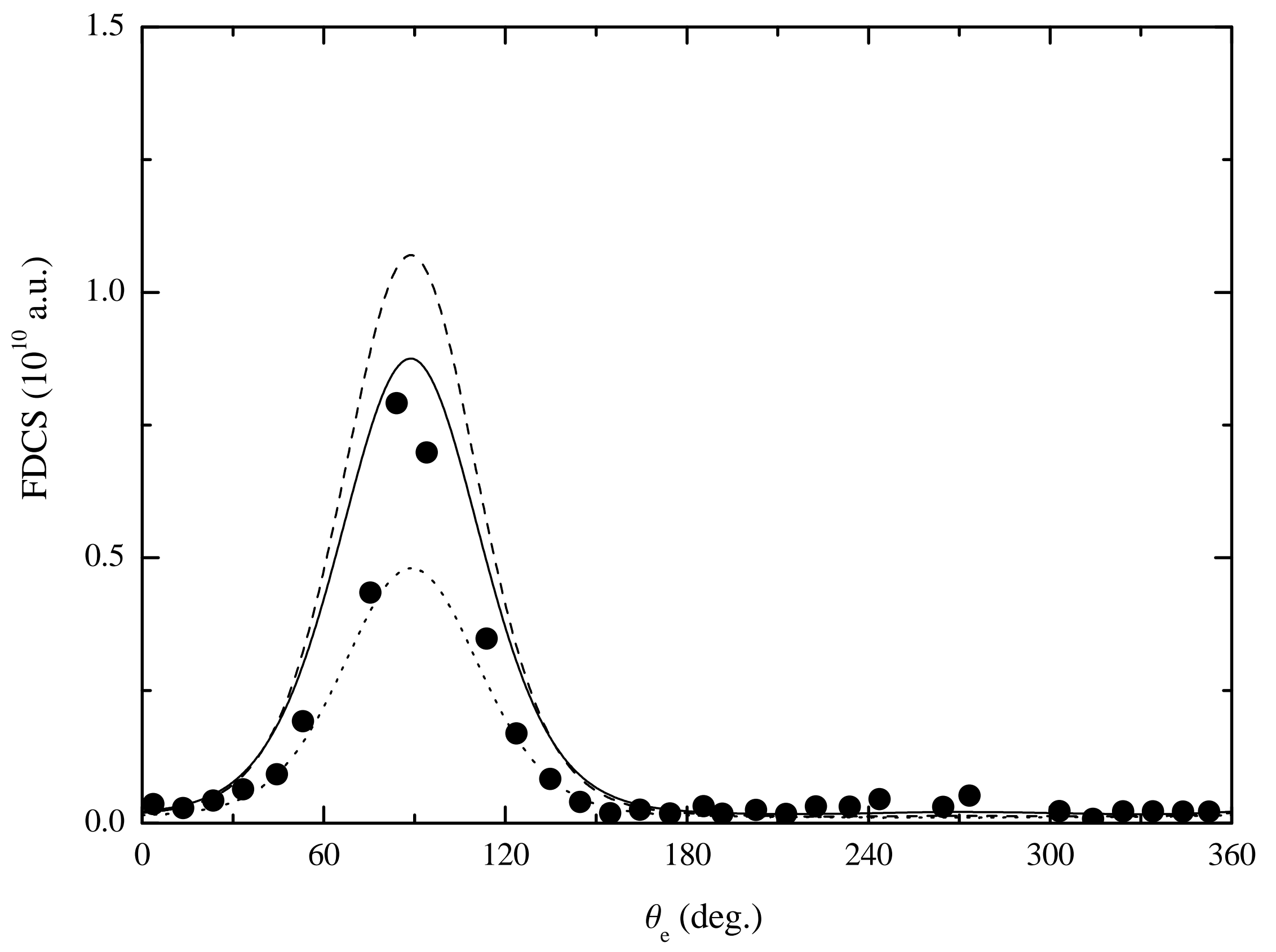




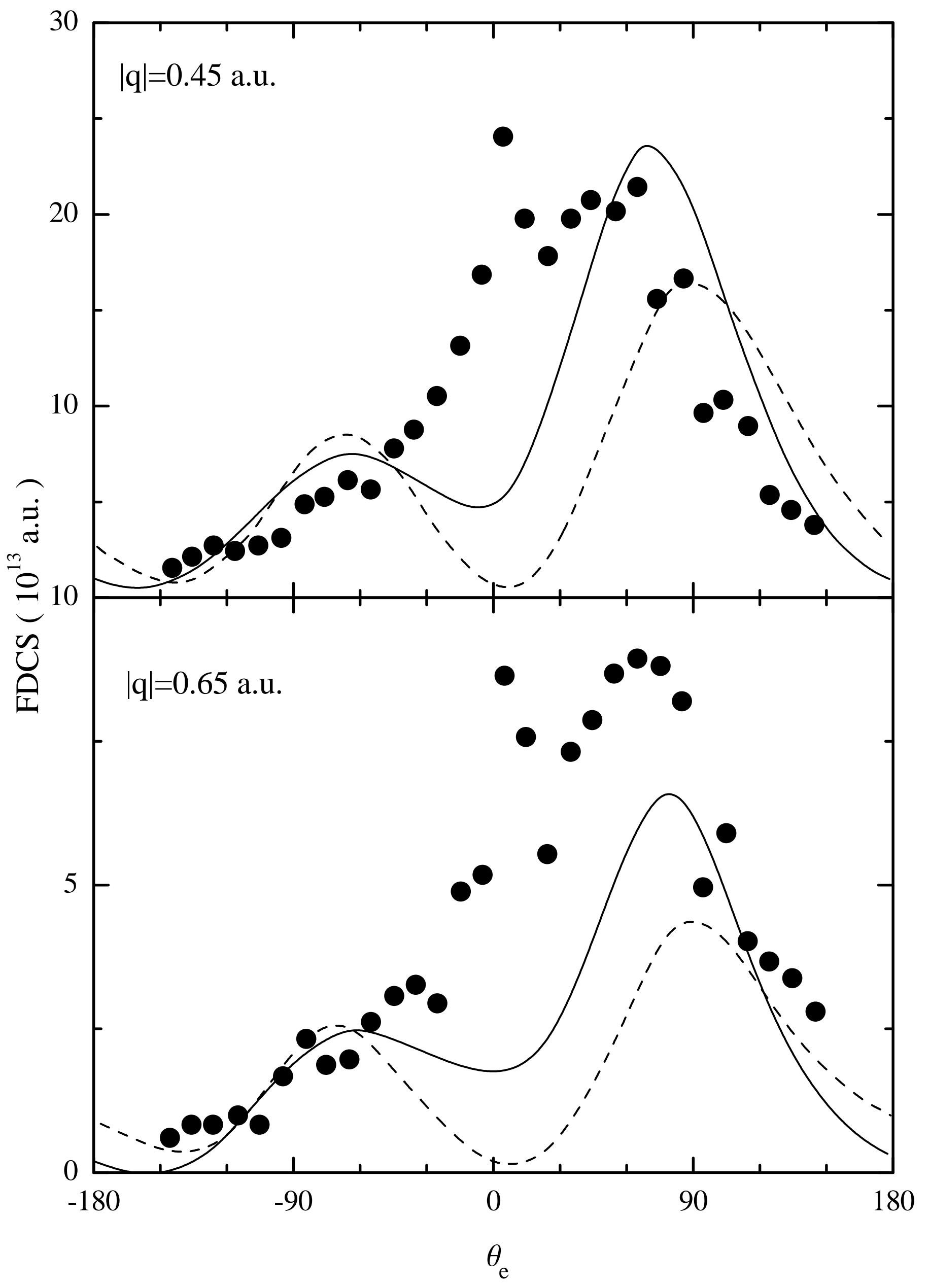




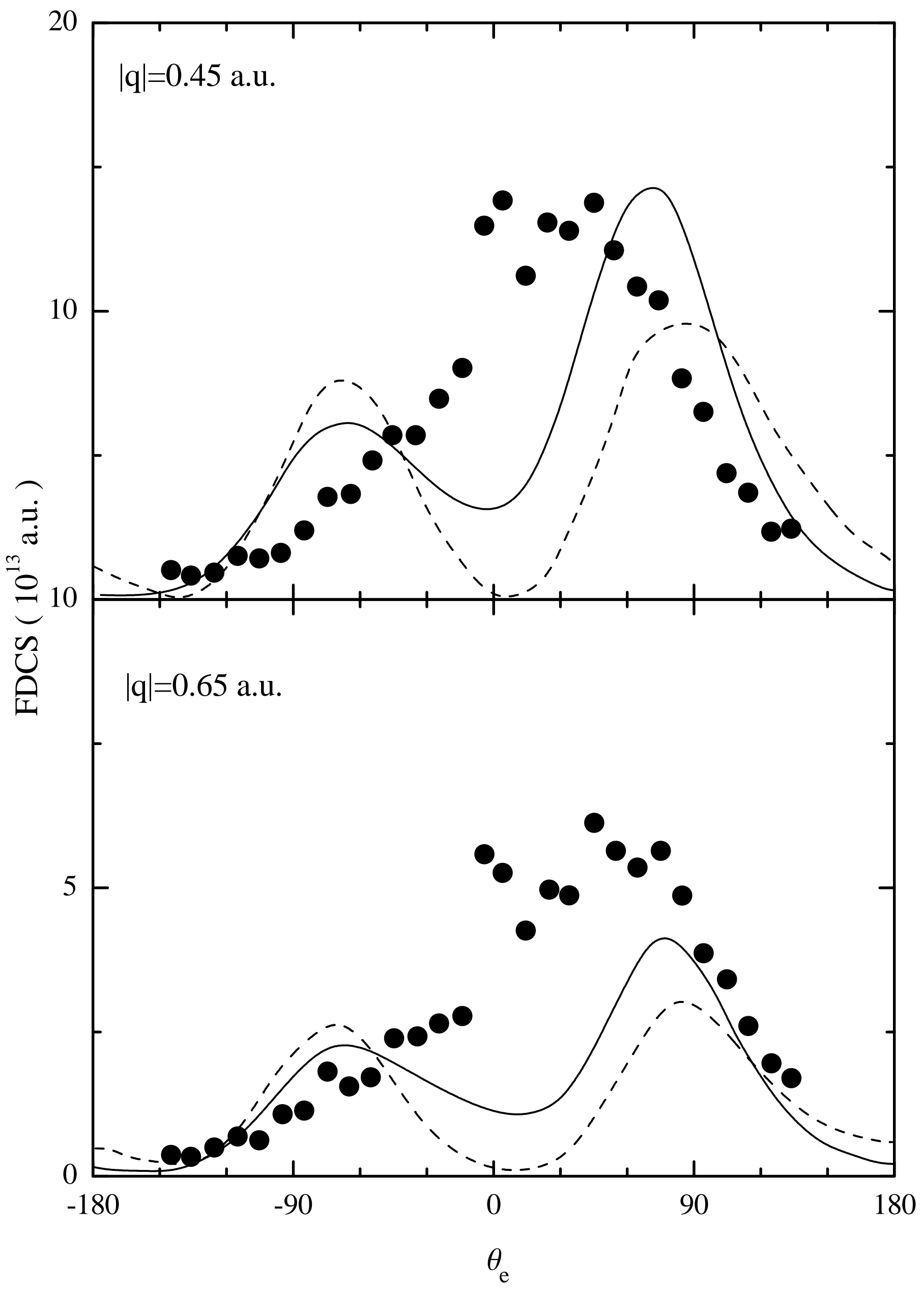




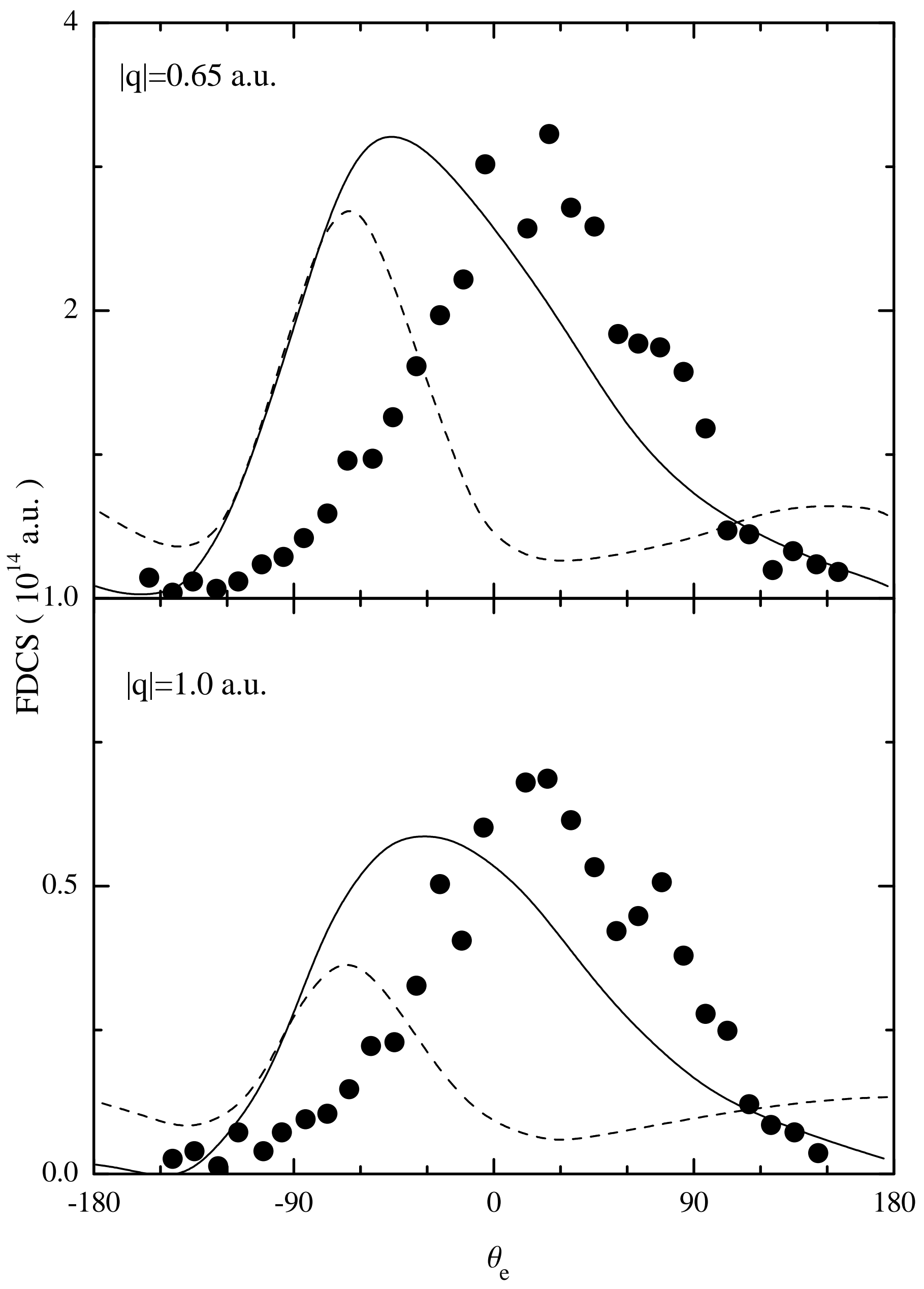




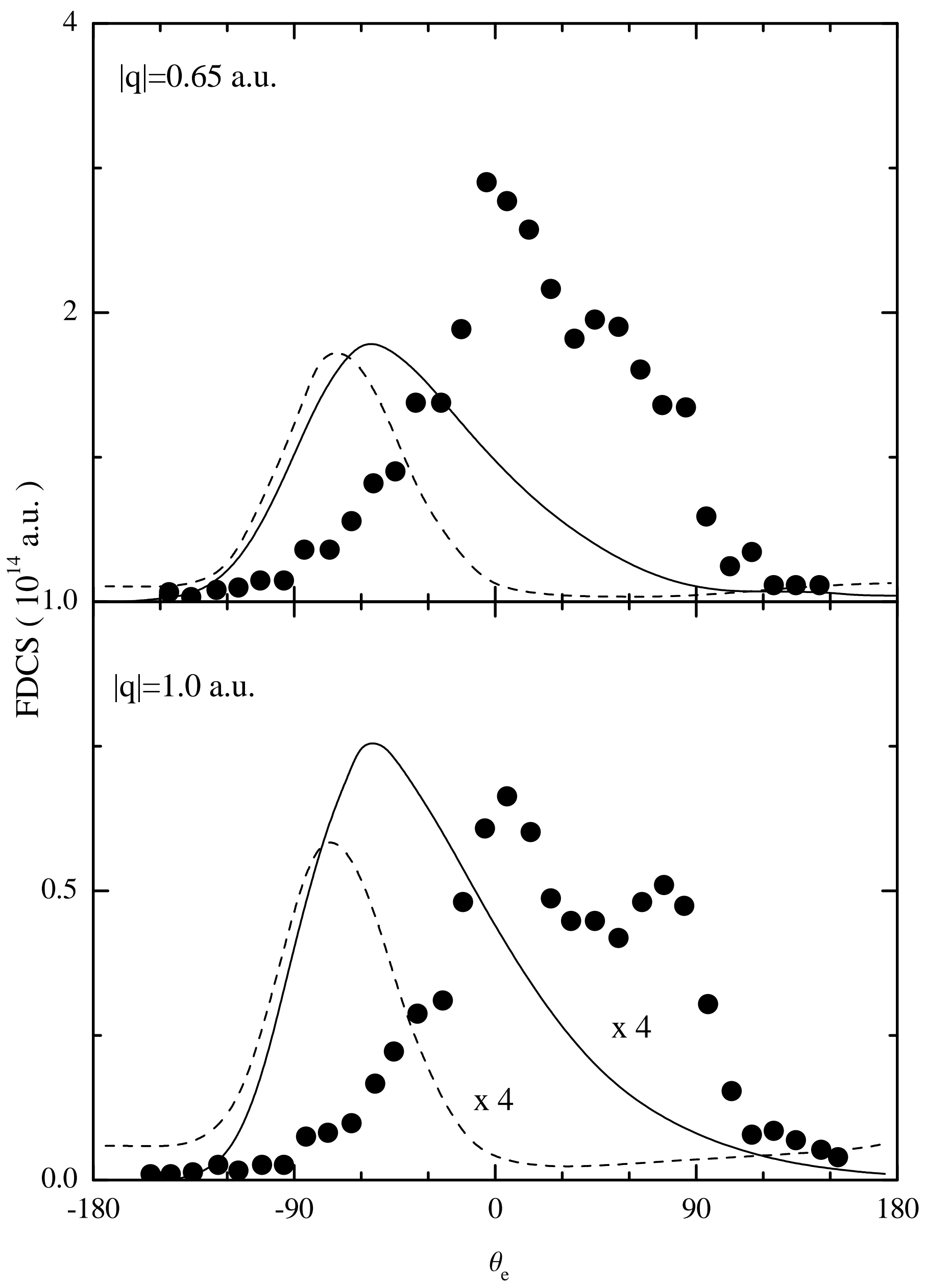

